\title{
Vibrational spectroscopy dissects a surface reaction
}

When a metal surface catalyzes a chemical process, each surface atom's local environment matters.

n 1909 Fritz Haber made a discovery that would change the world. Using a catalyst of solid iron mixed with a few other substances, he could break atmospheric nitrogen into its constituent atoms and combine them with hydrogen to produce ammonia, which in turn could be made into urea and other fertilizers. In the century that followed, agricultural productivity increased severalfold, and Earth's population became more food secure despite more than quadrupling. Although synthetic fertilizer wasn't the only factor to contribute to that improvement, it was an important one.

Industrial surface catalysis - now extended to many more reactions - is a messy process. The catalytic surfaces are rough and irregular, and hot reactant molecules jostle one another as they zip around in all directions. Catalysts and reaction conditions, therefore, have often been chosen mainly by trial and error, without much real understanding of how the reactions actually work.

To facilitate the rational design of new catalysts, chemical physicists typically start by simplifying the reactions: The craggy catalysts and pressurized reaction vessels in a chemical plant are replaced with smooth crystalline surfaces and rarefied atmospheres in the lab. Those experiments have yielded important findings, including the detailed mechanism of the Haber process, part of the body of work for which Gerhard Ertl was awarded a Nobel Prize in 2007 (see PHYSICS TODAY, December 2007, page 14, and the article by Ertl and Hans-Joachim Freund, PHYSICS TODAY, January 1999, page 32). But the need remains to better replicate the complexity of real-world conditions.

Rainer Beck, of the Swiss Federal Institute of Technology in Lausanne, and his colleagues are bridging the materials gap between lab and plant by studying the dissociative adsorption of methane on the (211) crystal face of platinum. Similar to industrial catalysts but unlike the (100) and (111) surfaces often used in lab experiments, $\operatorname{Pt}(211)$ 's surface sites aren't all equivalent. Previously, experiments could measure only the average reaction output of the whole surface. But earlier this year, Beck and company showed that they could separately probe the catalytic activity of each type of site. ${ }^{1}$

Now they've added a new layer of complexity. ${ }^{2}$ By replacing $\mathrm{CH}_{4}$ with deuterated methane, $\mathrm{CH}_{3} \mathrm{D}$, they've introduced the possibility of more than one dissociation reaction: The bond that breaks could be either C-H or C-D. Beck and colleagues disentangled the complicated relationship between reaction site, reactant speed, and reaction products to paint a picture of a surface reaction in unprecedented detail.

\section{Quantum control}

The new work is part of an ongoing effort to control every aspect of a surface reaction, including the kinetic energy

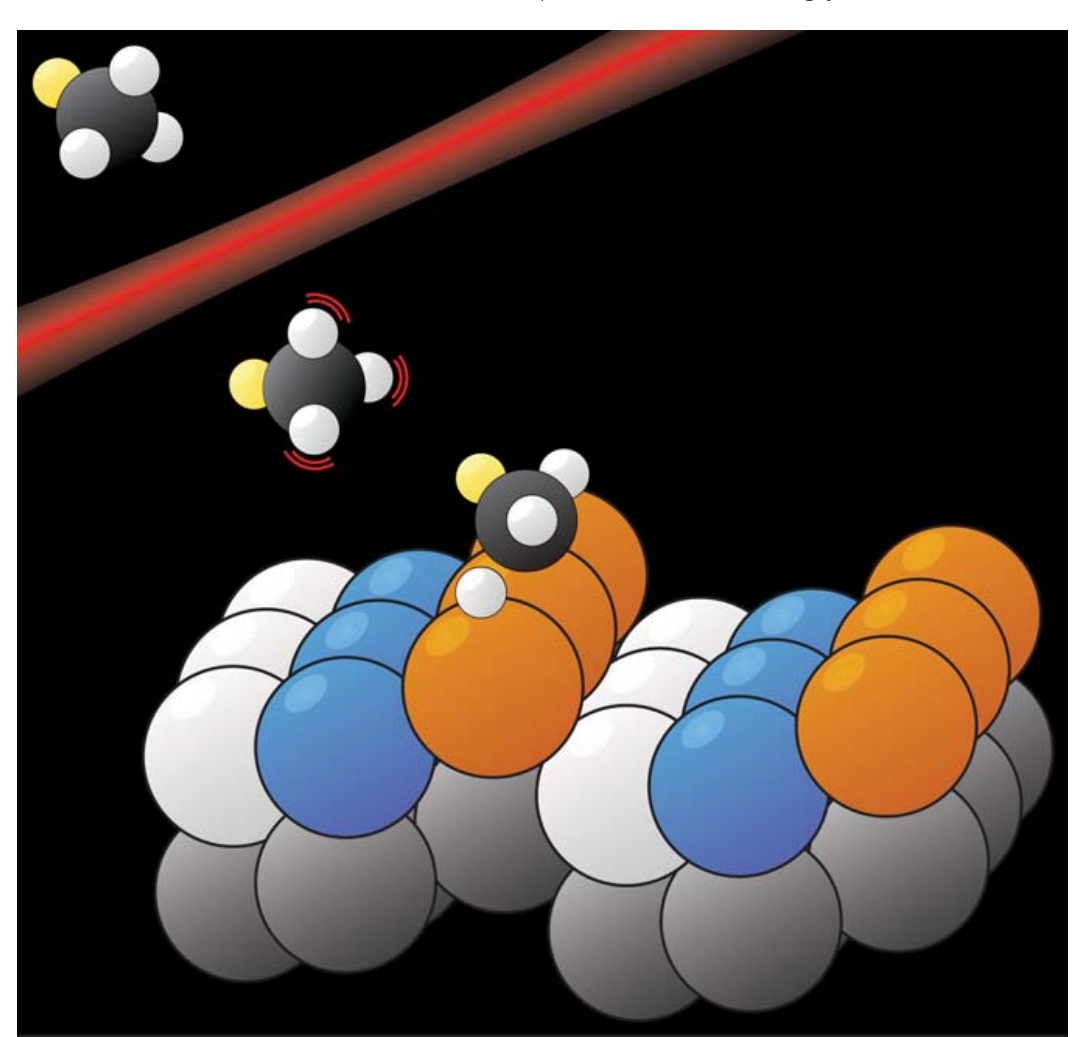

FIGURE 1. WHEN DEUTERATED METHANE, $\mathrm{CH}_{3} \mathrm{D}$, strikes the (211) surface of platinum, either a C-H or a C-D bond can break. Which bond breaks depends on the molecule's speed, quantum state, and whether the molecule is adsorbed on a step (orange) or terrace (blue) surface site. Passing the molecules through an IR laser beam (red) excites them to a vibrational quantum state with a preference for $\mathrm{C}-\mathrm{H}$ fission. (Courtesy of Rainer Beck.) 


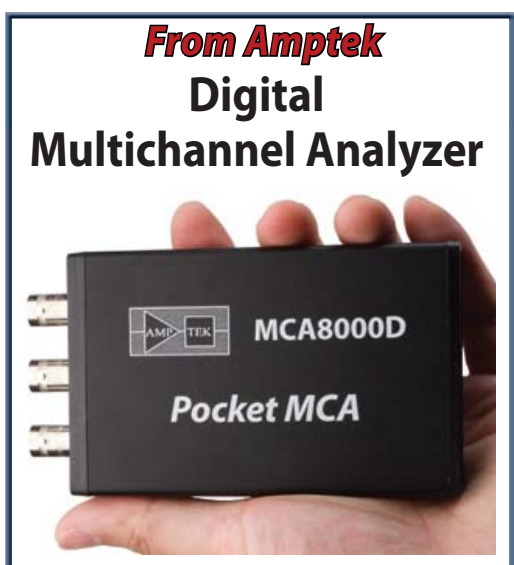

The MCA8000D is a full-featured digital multichannel analyzer intended to be used with a wide variety of detector systems.

The easy to use 'Pocket MCA' can fit in a shirt pocket.

\section{Features of the MCA8000D}

- Compatible with traditional analog pulse shaping

- High speed ADC (100 MHz, 16 bit) with digital pulse height measurement

- 8k data channels

- Minimum pulse peaking time $500 \mathrm{~ns}$

- Conversion time $10 \mathrm{~ns}$

- Sliding-scale linearization

- Differential nonlinearity $< \pm 0.6 \%$

- Integral nonlinearity $< \pm 0.02 \%$

- Two peak detection modes for nuclear spectroscopy or particle counter calibration in clean rooms.

- Two TTL compatible gates for coincidence and anticoincidence

- USB, RS-232, and Ethernet communication interfaces

- USB powered

- Dimensions: 5 x $2.8 \times 0.8$ in

- Weight: <165 g

\section{Free Software}

Free Software Development Kit (SDK). Complete protocol and example code for custom software applications.

Free Display and Acquisition software.

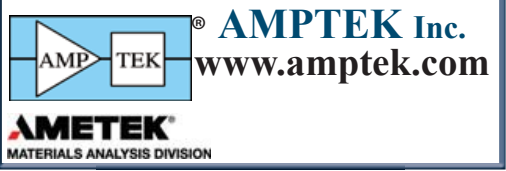

\section{SEARCH \& DISCOVERY}

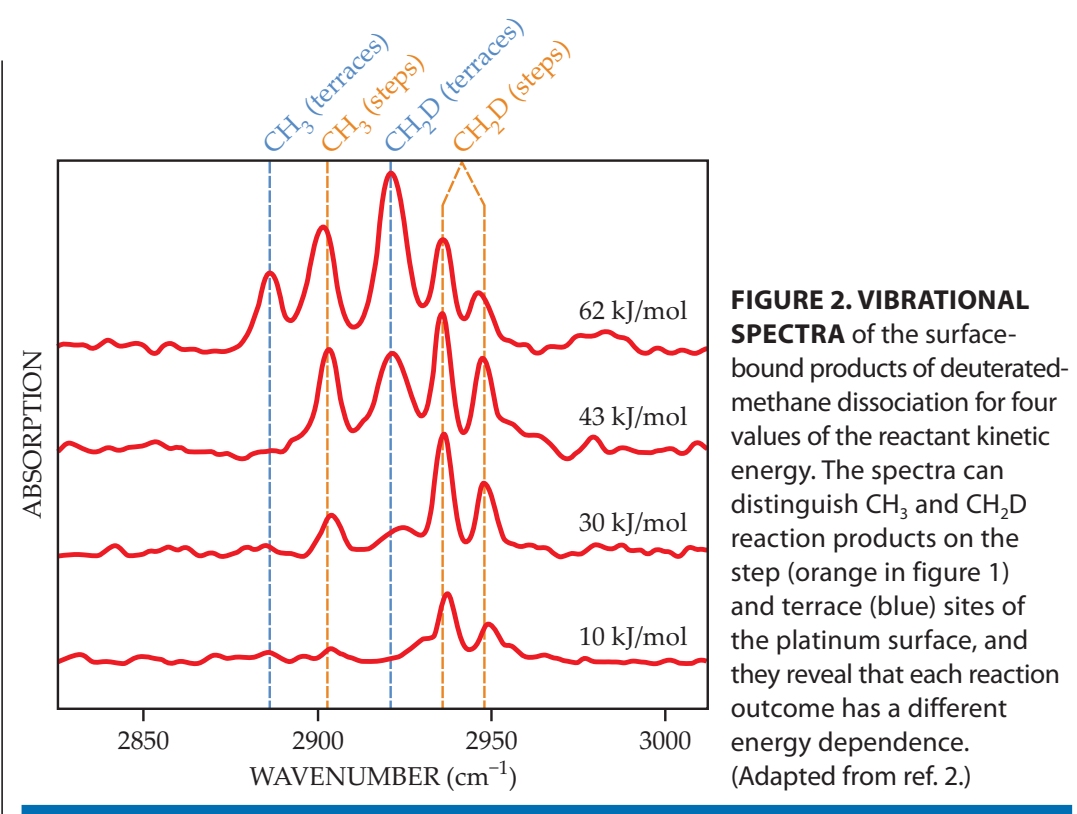

does not, the molecule exhibits a strong preference for $\mathrm{C}-\mathrm{H}$ bond dissociation. ${ }^{4}$

Such control over the outcome of a chemical process, although appealing, is not of any immediate practical consequence. Chemical plants aren't set up to use lasers to prepare their reactants in specific quantum states. But the results can offer important insights into how the reaction works.

One of the simplest ways to describe a chemical process is with statistical rate theory. In that approach, both the overall reactivity and the branching ratio between different reaction outcomes depend only on the reactant's total energy, not its specific quantum state. When statistical rate theory fails to describe a reaction well, more complicated models are required, and they'll need to be benchmarked with experimental data.

\section{Site resolution}

To monitor the surface reactions, Beck and company use reflection absorption IR spectroscopy (RAIRS), a nondestructive technique to identify surface-bound species from their resonant vibrational frequencies. The bonds of adsorbed $\mathrm{CH}_{3}$, for example, vibrate at different frequencies than those of $\mathrm{CH}_{2} \mathrm{D}$. And curiously, an experiment conducted by Beck's former postdoc Helen Chadwick showed that on $\mathrm{Pt}(211), \mathrm{CH}_{3}$ itself had a measurably different vibrational spectrum depending on where on the surface it was located. ${ }^{1}$ The $\mathrm{C}-\mathrm{H}$ stretching frequency on the terrace sites (blue in figure 1) is

\section{PHYSICS TODAY I SEPTEMBER 2018}

about $15 \mathrm{~cm}^{-1}$ lower than on step sites (orange); the corner sites (white) don't induce any measurable dissociation at all.

The precise reason for the frequency shift is a bit of a mystery. "We know that it's due to differences in the weakening, or softening, of the $\mathrm{C}-\mathrm{H}$ bonds," says Beck. "Apparently, the softening for $\mathrm{CH}_{3}$ is less strong on the steps than on the terraces." But that's not a general phenomenon. Carbon monoxide, in fact, shows the opposite effect, with higher vibrational frequencies on the terraces than on the steps. That difference remains unexplained.

Whatever the cause, the Lausanne researchers have put the shift to good use. By monitoring the vibrational spectrum of the whole surface and resolving it into site-specific components, they measured the catalytic activity of each type of site. Qualitatively, the results were no surprise: The more-exposed step sites are more active than the less exposed terrace sites. But the RAIRS experiments allowed the researchers to go beyond that simple description and study the sites in quantitative detail: measuring the energetic barrier to reaction on each type of site, for example, or looking for other dependencies on $\mathrm{CH}_{4}$ speed or quantum state that might not be obvious from surfaceaveraged measurements.

The power of site-resolved measurements is made clear in the new deuterated-methane experiments performed by Beck's student Ana Gutiérrez-González. ${ }^{2}$ Even when $\mathrm{CH}_{3} \mathrm{D}$ is prepared in a quantum state optimized for $\mathrm{C}-\mathrm{H}$ dissocia- 
tion, the product selectivity is not total, and its completeness depends on the kinetic energy of the molecular beam. On $\mathrm{Pt}(211)$, the steps and terraces each show their own energy-dependent product branching, as shown in figure 2. At the lowest kinetic energies, only the steps are catalytically active, and the vast majority of reactions are $\mathrm{C}-\mathrm{H}$ fission to form $\mathrm{CH}_{2} \mathrm{D}$. At progressively higher energies C-D fission on steps, C-H fission on terraces, and $\mathrm{C}-\mathrm{D}$ fission on terraces also contribute. At the highest energies, the branching ratio of $\mathrm{C}-\mathrm{H}$ to $\mathrm{C}-\mathrm{D}$ fission, deduced from the areas under the spectro- scopic peaks, approaches $3: 1$, the expected value for a nonselective reaction.

Figuring out what the results mean for the reaction mechanism will require an intense theoretical effort. The very fact that quantum states are important to the reaction outcome means that the dissociation can't be described by a simple model; rather, theoreticians must simulate the detailed quantum trajectories of the molecules striking the surface. The Lausanne experimenters are working with several theory groups to convert their results into more accurate reaction models, with the ultimate goal being a predictive understanding of surface reactions on complex catalysts. And to better mimic the complexity of industrial catalysts, they're extending their experiments to $\mathrm{Pt}(531)$, whose steps are zigzag shaped.

Johanna Miller

\section{References}

1. H. Chadwick et al., J. Chem. Phys. 148, 014701 (2018).

2. A. Gutiérrez-González, F. F. Crim, R. D. Beck, J. Chem. Phys., in press.

3. R. D. Beck et al., Science 302, 98 (2003); R. R. Smith et al., Science 304, 992 (2004). 4. D. R. Killelea et al., Science 319, 790 (2008).

\section{Semiconductor crystals achieve record thermal conductivity}

The synthesis of low-defect boron arsenide crystals could reduce overheating in electronic devices.

W hen a laptop or cell phone heats up from overuse, it's not just uncomfortable for the user. That excess heat also damages the circuitry and reduces the device's performance, energy efficiency, and life span. As modern electronic components are made smaller, their resistance rises. Heat dissipation has become a critical technological challenge for next-generation items, including microprocessors and integrated circuits, LEDs, and high-powered RF products.

Materials with high thermal conductivity help dissipate heat and improve the performance and reliability of those devices. However, developing a passive cooling option that is both cost-effective and reliable has been difficult. With a thermal conductivity of $2000 \mathrm{~W} / \mathrm{m} \cdot \mathrm{K}$, diamond is the best-known material for cooling. But it is expensive, has slow synthesis rates, and is of varying quality. Integrating diamond with silicon and other industrial semiconductors is also challenging because those materials have different thermal expansion coefficients.

Among metals, copper has the highest thermal conductivity at room temperature, $400 \mathrm{~W} / \mathrm{m} \cdot \mathrm{K}$, and is commonly used in industry for dissipating heat in electronics. Among binary compounds, silicon carbide, at $350 \mathrm{~W} / \mathrm{m} \cdot \mathrm{K}$, is favored for thermal management in personal electronics. To control the high-density heat

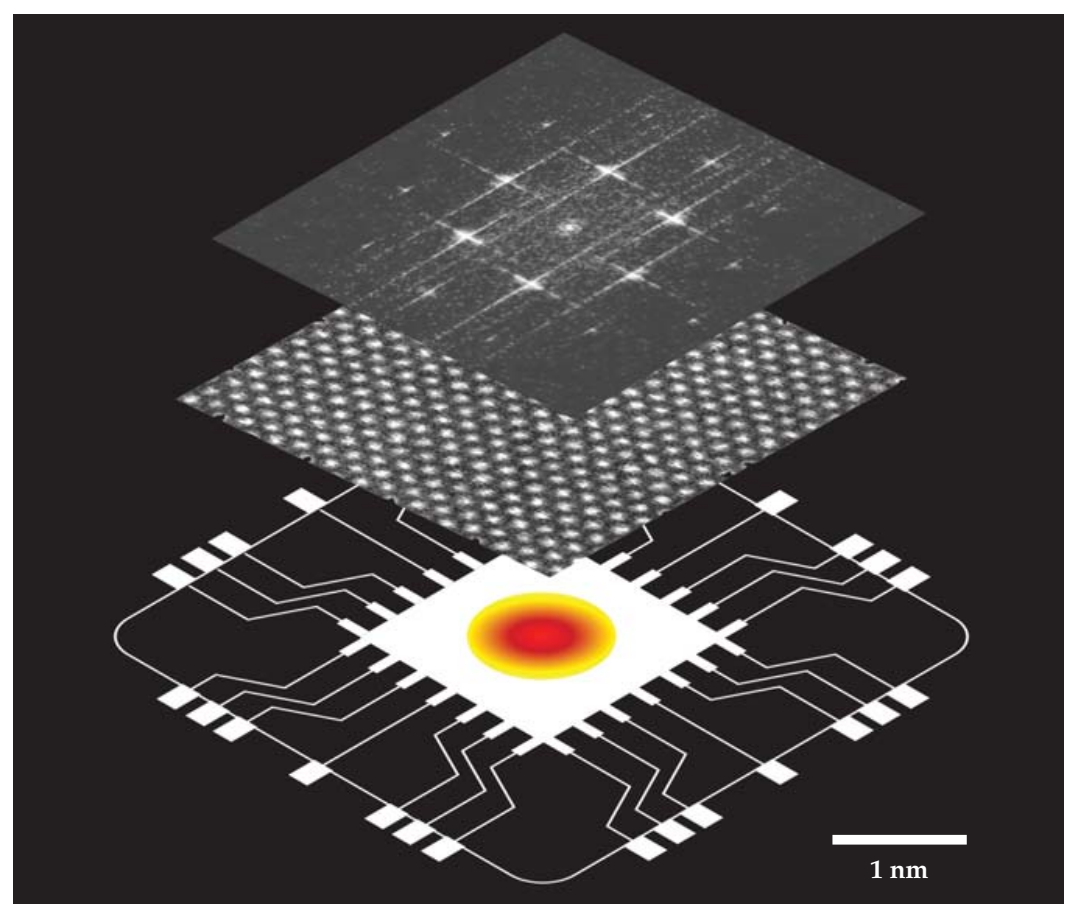

FIGURE 1. CONCEPT OF THERMAL MANAGEMENT AND A BORON ARSENIDE CRYSTAL. This illustration shows a computer chip with a hot spot at the center (bottom). A high thermal conductivity material like BAs could help dissipate the chip's heat. A transmission electron microscope image shows the atomically resolved lattices of a BAs crystal grown by UCLA researchers (middle). The electron diffraction pattern of the BAs crystal gives rise to clear bright spots, whose symmetry indicates the periodic structure and lack of defects in the crystal (top). (Image courtesy of Yongjie Hu.)

generated in small, power-hungry devices, however, the ideal material would have a thermal conductivity at least as high as diamond's.

Now three research groups have synthesized boron arsenide (BAs) crystals that have a thermal conductivity of more than $1000 \mathrm{~W} / \mathrm{m} \cdot \mathrm{K}$ at room temperature, exceeding that of all other materials except diamond. ${ }^{1-3}$ BAs still has a long way to go in terms of size, consistency, and cost before it could be used in an actual device. Nonetheless, the new research vindicates theoretical predictions 